\title{
Brain Abscess Caused by Parvimonas Micra with Successful Treatment: A
}

\section{Rare Case Report}

\section{Gonçalo Januário $^{1 *}$ | Roque Diaz² | Paul Nguewa3 ${ }^{3}$}

*Correspondence: Gonçalo Januário

Address: ${ }^{1}$ Centro Hospitalar Lisboa Central, Neurosurgical Department - Hospital de São José, Lisboa, Portugal; ${ }^{2}$ Hospital Garcia Orcoyen, Estella, Navarra, Spain; ${ }^{3}$ Universidad de Navarra, ISTUN Instituto de Salud Tropical. Department of Microbiology and Parasitology, Pamplona, Spain. Instituto de Investigaciones Sanitarias de Navarra (IdiSNA).

e-mail $\bowtie$ : jg.machado@hotmail.com

Received: 09 September 2021; Accepted: 16 September 2021

Copyright: (C) 2021 Januário G. This is an open-access article distributed under the terms of the Creative Commons Attribution License, which permits unrestricted use, distribution, and reproduction in any medium, provided that the original work is properly cited.

\begin{abstract}
Parvimonas Micra is an anaerobic gram-positive coccus, usually found in the human oral mucosa. We present a case of cerebral abscess provoked by Parvimonas Micra, an infrequent agent for this pathology, in an 85-year-old immunocompetent man with progressive onset left hemiparesis. The diagnosis was realized through the computed tomography and magnetic resonance imaging. Surgical options are aspiration or total removal of the abscess. Each of the options has associated advantages and disadvantages. In our case we decided to perform a craniotomy to the drainage of the pus and the removal of the capsule from the abscess. After the neurosurgical intervention and collection of purulent contents, the pathologic microorganism could be identified. For complement the treatment, antibiotics were administered. The patient's clinic clearly improved after the surgical and medical management, complemented by motor rehabilitation plan. The treatments applied to this patient were effective, with a progressively favorable evolution of the clinical status.
\end{abstract}

Keywords: Brain Abscess, Craniotomy, Parvimonas Micra

\section{Introduction}

\section{Epidemiology}

Brain abscesses are a purulent collection located in the brain parenchyma. They have an estimated incidence of 1 case per 10,000 hospitalized patients. There is a predominance in males, average age of presentation is between 30 and 45 years (Helweg-Larsen et al., 2012; Erdoğan and Cansever, 2008; Akashi et al., 2017).

The advent of computed tomography (CT) in the late 70s of the last century, the development of new surgical techniques and the availability of better antibiotics, allow a significant reduction in mortality caused by this pathology (currently between 5-20\%) as well as a lower incidence of neurological deficits associated with the injury itself. The rate of complications caused by surgical procedures has also been 
decreasing globally.

\section{Symptom and Signs}

The most frequent symptom is headache with an incidence of $70 \%$, fever and neurological deficits present in less than half of the cases. Seizures are diagnosed in $25-45 \%$ of cases. Papilledema and neck stiffness were seen in 25\% of patients (Helweg-Larsen et al., 2012; Erdoğan and Cansever, 2008; Akashi et al., 2017).

Although in $15-30 \%$ of cases there is no predisposing factor, the two main mechanisms at the origin of a brain abscess are hematogenous dissemination or by the extension of an adjacent focus. In the latter case, the origin is related to a contiguous infectious focus in the frontal sinuses, in the auditory system or in the dental arch (Helweg-Larsen et al., 2012; Erdoğan and Cansever, 2008; Akashi et al., 2017).

Laboratory tests are nonspecific, namely the study of cerebrospinal fluid, which generally shows negative results. In addition and due to the risk of herniation, lumbar puncture is usually contraindicated. In the case of blood cultures they present a positive result between 10-20\%, especially when the presence of ventriculitis is confirmed after a massive rupture of the abscess to the ventricular system.

In order to the adequate isolation of aerobic and anaerobic microorganisms, the material collected during the surgical procedure must be immediately processed in an appropriate culture medium (Akashi et al., 2017).

The initial diagnostic bases for brain abscess are the detailed clinical history and imaging techniques, namely simple tomography and especially after administration of contrast that enhances the abscess wall especially in its last stage of formation and also magnetic resonance imaging (MRI) after gadolinium. Both imaging techniques allow to identify the different stages of development of the brain abscess. In the case of MRI, the gadolinium contrast image, and the diffusion study, provide very relevant information (Kastrup et al., 2005).

Diffusion-weighted images (DWI) reveal an increased magnetic resonance signal with hyperintensity in the central portion of the abscess, suggesting the presence of decreased diffusion of water molecules. Other advanced MRI techniques such as spectroscopy and perfusion, may also be useful for additional information (Kastrup et al., 2005).

However, and regardless of the imaging technique used, they are not $100 \%$ sensitive or specific for the diagnosis of this pathology. Hence the importance of providing material for conducting the microbiological study to achieve a definitive diagnosis (Kastrup et al., 2005). 


\section{Current Treatment Hurdles}

The treatment of brain abscesses should be started as soon as possible after diagnosis, with a combined and multidisciplinary approach.

When indicated, a surgical procedure should be performed for the treatment of this pathology. There are several possible surgical approaches. Regardless of the technique used, it must allow the collection of material for the microbiological study and the consequent identification of the causative agent, as well as the decompression with reduction of the mass effect caused by the injury (Carson and Miulli, 2017; Longo et al., 2019; Chughtai et al., 2019).

Initially, the use of broad spectrum antibiotics is recommended, after the identification of the agent, the antibiotic treatment should be directed to the identified microorganism (Mombelli et al., 2017).

We describe a clinical case of brain abscess caused by Parvimonas Micra, a gram positive anaerobic coccus, a rare causal agent of this pathology (Baghban and Gupta, 2016).

\section{Case Report}

We present a case about a 85-year-old man, immunocompetent, with a previous clinical background of hypertension (HBP), dyslipidemia (DLP), chronic renal failure (CRF) and benign prostatic hypertrophy (BPH), without history of known dental pathology. Came to our hospital (Centro Hospitalar Lisboa Central, Neurosurgical Department - Hospital de São José, Lisbon, Portugal). He was treated with nifedipine (orally $30 \mathrm{mg} /$ day), olmesartan (40mg/day, orally), hydrochlorothiazide (12.5 mg/day, orally) and simvastatin $(20 \mathrm{mg} /$ day, orally. He came to the emergency room of our hospital exhibiting a progressive loss of strength in left limbs, drowsiness and confusion of several days of evolution.

In the general examination, the patient was conscious and collaborative, disoriented in time and space, and isochoric and photoreactive pupils. Along the neurological examination a hemiparesis was detected in the left side of the body of brachial predominance and in the lower limb, with associated homolateral facial component, without alteration of surface sensitivity and showing tendon reflexes (ROT) present and symmetrical, and an extensor left cutaneoplantar reflex.

Under the suspicion of a brain injury, an urgent CT image was performed showing a heterogeneous hypodense lesion in right frontoparietal region, and a perilesional vasogenic edema with mass effect causing moderate displacement of midline structures. We also observed an image of diffuse brain atrophy without intracranial acute hemorrhagic lesions and permeable skull's base cisterns. 
Magnetic resonance imaging (MRI) confirmed the presence of heterogeneous hypodense lesion in fronto-parietal region associated to perilesional edema, causing mass effect (Fig. 1).

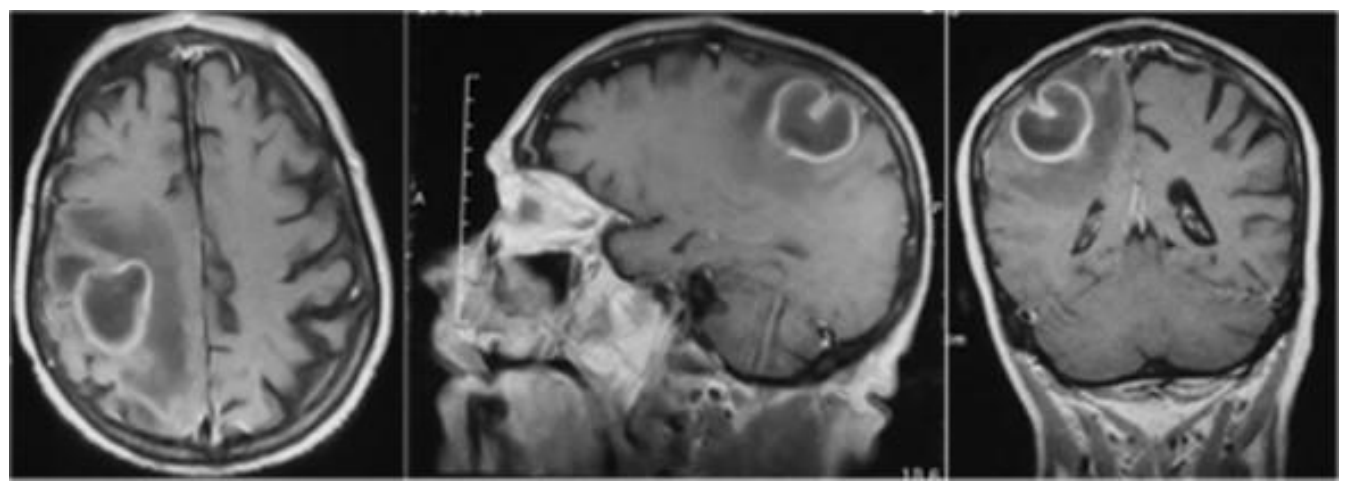

Figure 1: MRI T1 (axial; sagital; coronal) lesion, in right fronto-parietal topography, central cavity appears in as hypointense compared to parenchyma with perilesional vasogenic edema.

The central cavity appeared in $\mathrm{T} 1$ as hypointense in relation to the surrounding cerebral parenchyma and hyperintense in relation to the CSF of the ventricular system. Ring enhancement in the sequences obtained with paramagnetic contrast was observed. The most characteristic finding was the presence of a hypointense halo on $\mathrm{T} 2$ in the margin of the lesion in the marginal region corresponding to the capsule (Fig. 2).
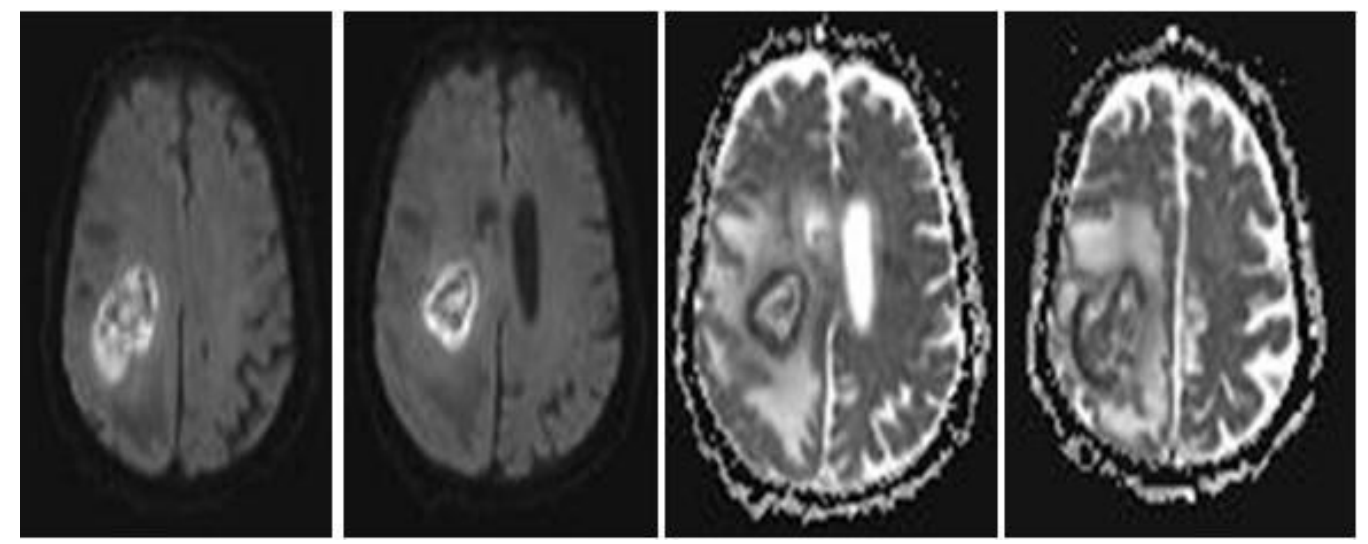

Figure 2: MRI DWI and ADC map (axial) lesion with diffusion restriction in the right cerebral hemisphere. Abnormalities compatible with abscess.

In the emergency area, he underwent a blood test. It showed normal values in the three cellular series (hemoglobin $10.4 \mathrm{~g} / \mathrm{dL}$; mean corpuscular volume: $99.2 \mathrm{fl}$; total leukocytes: 8,600/mm ${ }^{3}$ [88\% polymorphonuclear] and platelets $158,000 / \mathrm{mm}^{3}$ ). Through the coagulation study all values was normal. In the tests of renal function and electrolytes, the following results were obtained: urea $127 \mathrm{mg} / \mathrm{dL}$, creatinine $1.54 \mathrm{mg} / \mathrm{dL}$, sodium $135 \mathrm{mg} / \mathrm{dL}$; potassium $4.6 \mathrm{mg} / \mathrm{dL}$; chlorine $101 \mathrm{mg} / \mathrm{dL}$, glucose $139 \mathrm{mg} /$ $\mathrm{dL}$ ). The analytical study of liver function were normal (total bilirubin $0.51 \mathrm{mg} / \mathrm{dL}$; Lactate EU $183 \mathrm{U} / \mathrm{L}$; 
aspartate aminotransferase $28 \mathrm{U} / \mathrm{L}$; alanine aminotransferase $20 \mathrm{U} / \mathrm{L}$, creatine kinase $310 \mathrm{U} / \mathrm{L}$, and pancreatic amylase $21 \mathrm{U} / \mathrm{L}$ ). The level of C-reactive protein was $350.6 \mathrm{mg} / \mathrm{L}$ a marker of acute phase reactant, supported the hypothesis that an infection may occur.

In the same emergency room, an electrocardiogram (ECG) was performed showing a sinus rhythm $70 / \mathrm{pm}$ without alterations in repolarization. The simple chest radiography did not show alterations in the lung parenchyma. There was no pleural effusion, no deviation of mediastin, and no other acute alterations.

The worsening of his neurological status together with fever and vomiting, as well as CT and MRI images were data consistent with brain abscess.

Several studies have evaluated the ideal treatment for brain abscesses. They recommend performing surgery in lesions larger than $2.5 \mathrm{~cm}$ and surgically accessible, associated with antibiotics. First empirically with broad-spectrum antibiotics and posteriorly according to the antibiogram of the isolated agent, if exchange is recommended. Some recent studies show good results with stereotactic aspiration especially for those with dimensions less than $1 \mathrm{~cm}$ and deep location (Murdoch, 1998).

Given surgical indications based on the age of our patient, the neurological status and image data, he underwent a right parietal craniotomy centered to the lesion, draining abundant purulent content, with partial removal of the abscess capsule. Samples were collected for microbiological studies (aerobic and anaerobic bacteria, mycobacteria and fungi) and histological analyses.

Through the histological studies, five brown fragments were obtained, showing a elastic consistency; fragments of 2-5 mm long were extracted. Microscopically the fragments were infiltrated by numerous granulocytes, neutrophils and histiocytes, compatible with abscess. No neoplastic tissue was identified.

The extracted material was also sent to the laboratory of microbiology. Gram staining did not reveal the presence of microorganisms. After 48 hours of cultivation in aerobic media, no bacterial growth was observed resulting sterile crops. Interestingly, cultures in anaerobic media were positive for Parvimonas Micra.

Blood cultures performed in urgency and before started the antibiotics were negative.

The studies of mycobacteria and fungi were negative for both. Therefore, the patient was treated with vancomycin $1000 \mathrm{mg}$, ceftriaxone $1000 \mathrm{mg}$ and metronidazole $15 \mathrm{mg} / \mathrm{ml}$ in the day of the surgery and during 6 weeks. As recommended the antibiotics should be initiated as early as possible. Initially with 
broad-spectrum antibiotics, subsequently adjusting to the antibiogram of drug sensitivity/resistance.

In the postoperative CT image, the patient exhibited a subtotal absorption of intraparenchymal cortico-subcortical hematological densities in the area right parietal convexity. In addition, a reduced vasogenic edema with re-expansion of the cortical sulci in the cerebral hemispheres was observed (Fig. 3). The patient had no symptoms at discharge and along subsequent controls, during follow-up, he presented good clinical and imaging evolution.
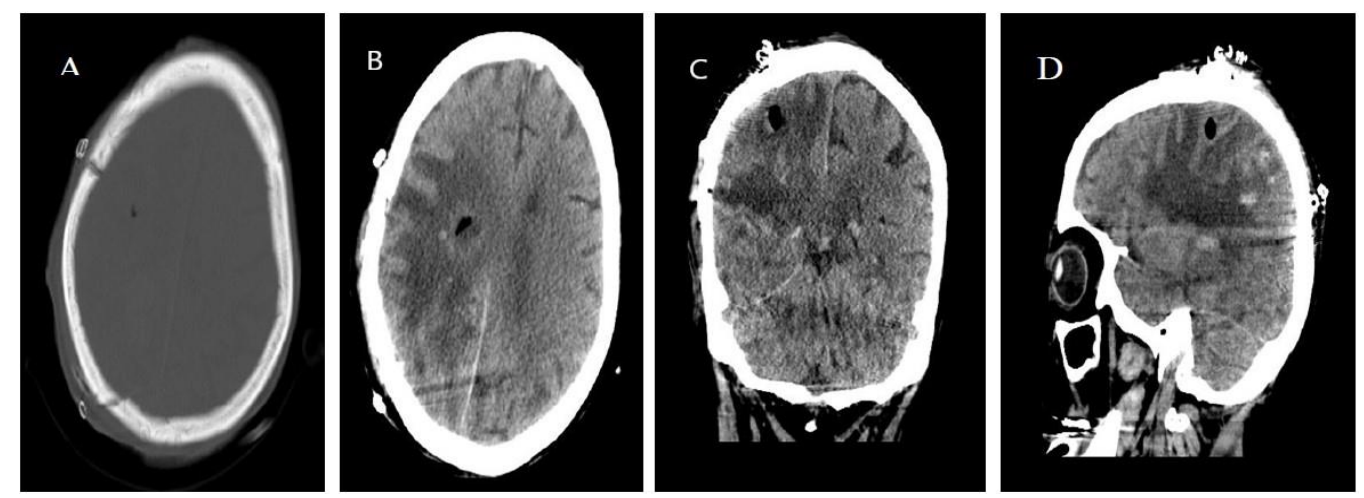

Figure 3: Postoperative CT images; At the left, axial slices, (A) bone and (B) parenchymal window. Right side, (C) coronal and (D) sagittal slices. No major complications present.

\section{Discussion}

Parvimonas Micra is a non-spore forming anaerobic gram-positive coccus (Baghban and Gupta, 2016). It is usually present and widely present in normal human oral and gastrointestinal flora. The predisposing factors associated with the occurrence of this infection are immunodeficiency, diabetes mellitus, chronic treatment with corticosteroids, history of previous surgery and neoplasia (Baghban and Gupta, 2016).

In these conditions, these bacteria can cause opportunistic infections in the central nervous system, thorax, abdomen, and pelvis, as well as generalized necrotizing soft tissue infections. Opportunistic infections caused by P. micra usually cause brain abscesses and endocarditis after dental manipulations. Within the thorax, P. micra may induce pneumonia, lung and cerebral abscesses, empyema, and mediastinitis. Rarely this agent causes infections in patients without recognized predisposing factors.

However, when dealing with cases such as the current, the treatment could be shortened with a proper surgical drainage.

Craniotomy, craniectomy or a simple burr hole are neurosurgical procedures performed for the treatment of a wide variety of intracranial pathologies. Postoperative tomography is important to exclude 
complications such as hemorrhage, hypertensive pneumocephalus or infections.

All surgical techniques are associated with complications, in the case of the techniques previously mentioned, the highest rates are related to craniectomy, namely the trephine syndrome (Brouwer et al., 2014). This technique is considered more aggressive because of the changes it causes and which are related to the complications that are inherent to it. A few months after craniectomy its necessary perform a cranioplasty to achieve a balance of intra and extracranial pressures, reducing the symptoms associated with trephine syndrome (Brouwer et al., 2014).

In our experience the most common approach are craniotomy and burr hole, craniectomy is reserved for only a few cases when exuberant cerebral edema is observed intraoperatively. This approach is in line with that described in the published series on this pathology. Craniotomy present more comorbidity if compared with burr hole but also higher removal rate with less recurrence. This is due to the fact that through the burr hole we have less access to aspirate or drain the pus. Burr hole approach are recommended to deep abscess like a thalamic abscess should be drained by a guided stereotactic procedure (Brouwer et al., 2014).

Craniotomy is necessary and recommended in selected cases, is the procedure of choice for cerebellar abscesses (Brouwer et al., 2014).

Initially in all cases, broad-spectrum intravenous antibiotic therapy should be administered, after identifying the agent, treatment adjusts to the microorganism for a minimum of 6-8 weeks to prevent recurrence (Longo et al., 2019; Brouwer et al., 2014; Cantiera et al., 2019).

The long term outcome is gratifying if prompt treatment is instituted in appropriate time period.

Before abscess encapsulation and localization, antimicrobial therapy, accompanied by measures to control increasing intracranial pressure, are essential. Once an abscess has formed, surgical excision or drainage combined with prolonged antibiotics (usually 4-8weeks) remains the treatment of choice (Cantiera et al., 2019).

\section{Conclusion}

A cerebral abscess is a collection of pus with intracranial location that reaches the surrounding brain parenchyma. The incidence of brain abscesses is higher in western countries and in males.

The early diagnosis of these injuries is a basic factor in the choice of treatment and the multidisciplinary approach is fundamental for the successful management of this pathology, it must be 
treated in an emerging way. In abscesses with a diameter greater than $2 \mathrm{~cm}$, surgical intervention is recommended using the technique that best fits each case. The surgical treatment must be supplemented with antibiotics.

Despite advances in diagnostic techniques and treatment, brain abscess remains a challenging clinical problem with substantial case fatality rates. Delays in diagnosis and treatment can result in a poor outcome (Brook, 2017).

Once an abscess has formed, surgical excision or drainage combined with prolonged antibiotics (usually 4-8weeks) remains the treatment of choice (Cantiera et al., 2019).

Significant advent in brain imaging, minimally invasive surgery, molecular biology, and antibacterial agents, has dramatically improved the prognosis. Main indicators of outcome include altered mental status at presentation and intraventricular rupture (Cantiera et al., 2019).

Most cases have a good clinical and imaging response, as in the case described, but we can always improve the current results.

Conflict of Interests: The authors don't have nothing to declare.

Acknowledgements: Thank you to Antonio Medarde (M.D., PhD) and Miguel Laia (M.D.) for his valuable comments.

\section{References}

Akashi M, Tanaka K, Kusumoto J, Furudoi S, Hosoda K, Komori T. Brain abscess potentially resulting from odontogenic focus: report of three cases and a literature review. J Maxillofac Oral Surg 2017; 16: 58-64.

Baghban A and Gupta S. Parvimonas micra: A rare cause of native joint septic arthritis. Anaerobe 2016; 39: 26-27.

Brook I. Microbiology and treatment of brain abscess. J Clin Neurosci 2017; 38: 8-12.

Brouwer MC, Tunkel AR, McKhann GM, van de Beek D. Brain Abscess. N Engl J Med 2014; 371: 447-456.

Cantiera M, Tattevin P, Sonneville R. Brain abscess in immunocompetent adult patients; Rev Neurol (Paris) 2019; 175: 469-474.

Carson T and Miulli D. Bedside twist drill aspiration of cerebral abscess less than $2.5 \mathrm{~cm}$ in size: A case series and discussion. Surg Neurol Int 2017; 8: 230.

Carson T and Miulli D. Bedside twist drill aspiration of cerebral abscess less than $2.5 \mathrm{~cm}$ in size: A case series and discussion, Surg Neurol Int 2017; 8: 230.

Chughtai KA, Nemer OP, Kessler AT, Bhatt AA. Post-operative complications of craniotomy and craniectomy. Emerg Radiol 2019; 26: 99-107. 
Erdoğan E and Cansever T. Pyogenic brain abscess. Neurosurg Focus 2008; 24: E2.

Helweg-Larsen J, Astradsson A, Richhall H, Erdal J, Laursen A, Brennum J. Pyogenic brain abscess, a 15 year survey. BMC Infect Dis 2012; 12: 332 .

Kastrup O, Wanke I, Maschke M. Neuroimaging of infections. NeuroRx 2005; 2: 324-332.

Longo M, Feigen C, De la Garza Ramos R, Gelfand Y, Echt M, Agarwal V. Predictors of reoperation and noninfectious complications following craniotomy for cerebral abscess. Clin Neurol Neurosurg 2019; 179: 55-59.

Mombelli A, Almaghlouth A, Cionca N, Cancela J, Courvoisier DS, Giannopoulou C. Microbiologic Response to Prediction Therapy and Multivariable Prediction of Clinical Outcome. J Periodontol 2017; 88: 1253-1262.

Murdoch DA. Gram-Positive Anaerobic Cocci. Clin Microbiol Rev 1998; 11: 81-120.

Muzumdar D, Jhawar S, Goel A. Brain abscess: An overview. Int J Surg 2011; 9: 136-144. 